\title{
Neurological involvement in X-linked hypophosphataemic rickets
}

\author{
P G BRADBURY, D P BRENTON, G M STERN \\ From University College Hospital, London, UK
}

SUMMARY X-linked hypophosphataemic rickets is a familial form of Vitamin D resistant rickets in which gross bony and ligamentous changes may occur. Two patients showing severe spinal disease with evidence of spinal cord compression requiring neurosurgical intervention are reported. The management of such lesions may be problematic as cord compression may be found at several levels at presentation, and further difficulties develop after neurosurgical treatment.

$\mathrm{X}$-linked hypophosphataemic rickets is characterised by (1) X-linked dominant pattern of inheritance, (2) decreased renal tubular reabsorption of inorganic phosphate, (3) resistance to treatment with calciferol or cholecalciferol, (4) an absence of proximal myopathy, (5) a probable reduced formation of 1,25 dihydroxycholecalciferol in the renal tubule. ${ }^{1}$

Vitamin D resistant rickets was first described by Albright $e t$ al. $^{2}$ Christensen ${ }^{3}$ later described familial Vitamin D resistant rickets occurring in a mother, son and daughter. Initially an autosomal dominant inheritance was proposed but later an X-linked dominant mode of inheritance was demonstrated. The disease is regarded as a genetic defect of renal tubular phosphate transport but the relationship of that defect to the impaired formation of 1,25 dihydroxycholecalciferol is unclear and the cause of the ligamentous calcification is unexplained.

In its mildest form, the only abnormality may be the hypophosphataemia. Bony abnormalities, when present, are always relatively severe in males. Females may show a spectrum from absent bone disease to changes as severe as the males. Typically affected adults are of short stature with deformed legs and other typical rachitic bony changes. The skull may be deformed and there may be maxillo-facial abnormalities in particular.

Address for reprint requests: Dr P Bradbury, The National Hospital for Nervous Diseases, Queen Square, London WCIN 3BG, UK.

Received 8 July 1986 and in revised form 25 September 1986. Accepted 27 September 1986
In some adults there is active osteomalacia with pseudo-fractures and raised alkaline phosphatase. Serum calcium is normal but the urinary calcium excretion may be normal or low. Parathormone levels are normal or high. Secondary hyperparathyroidism is uncommon, however, unless high oral phosphate treatment has depressed the plasma calcium. Parathyroid adenomas are very rare. Bony overgrowth at sites of major muscle attachment and around joints may cause significant limitation of movement particularly at the elbows, shoulders and hips. There may be associated muscle atrophy, weakness and pain. The EMG and serum CK are normal.

We present two cases with severe bony spinal disease and spinal cord compression. Their clinical and biochemical features are summarised in the table.

Table Clinical and biochemical features in Cases 1 and 2

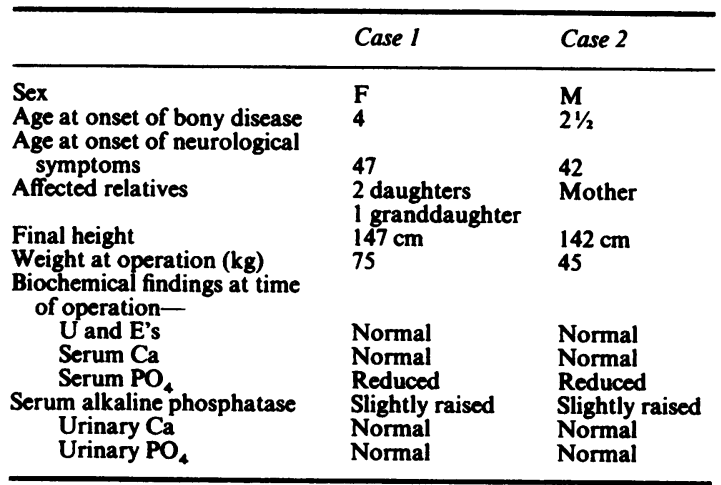



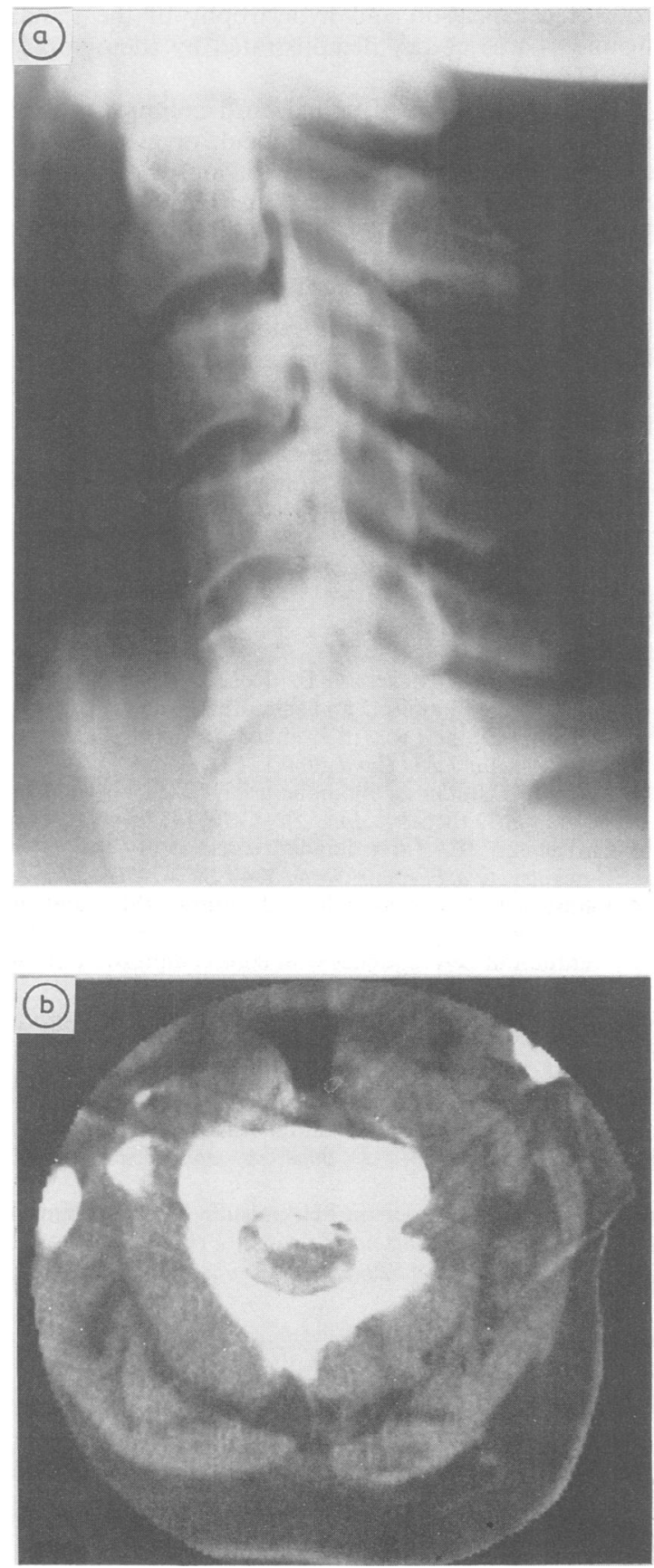

Fig (A) Tomogram of cervical spine showing hyperostosis of the posterior longitudinal ligament with narrowing of the spinal canal. (B) CT scan of cervical spine showing calcification and hypertrophy of posterior longitudinal ligament with thickened vertebral laminae.

\section{Case 1}

A female now aged 55 years, first presented to this hospital in 1973, hypophosphataemic rickets having been diagnosed in childhood. Bilateral osteotomies of the hips and knees had been performed at the age of 7 years. When first seen she was of short stature $(4 \mathrm{ft} 10 \mathrm{ins}, 147 \mathrm{~cm})$ with genu varus deformities of her legs. The patient had two affected daughters and one affected granddaughter. In 1976 at the age of 47 she complained of intermittent tingling in the fingers of both hands and both feet. Tomograms of the cervical spine performed at that time showed hyperostosis of the posterior longitudinal ligaments with narrowing of the cervical canal (fig, a). The patient's condition improved spontaneously and no further action was taken.

Three years later, whilst attending a magicians' conference in the USA, a friend greeted her with a "bear hug". Immediately she developed severe pain around the lower thorax, sagged on to her knees and lost all feeling in her legs. For the rest of the day she was unable to walk without support. She returned to this country with a spastic paraparesis with a sensory level between D7 and D10. Two initial attempts at myelography were unsuccessful owing to calcification of the spinal ligaments. At the third successful attempt the theca was found to be compressed in its anterior aspect by a calcified longitudinal ligament between $\mathrm{C} 3$ and $\mathrm{C5}$, but more significantly, partial obstruction of the dorsal theca at D7/D8 level due to constriction from the posterior aspect was demonstrated. A dorsal laminectomy was performed at which the laminae were found to be thickened and a calcified ligamentum flavum was found to be indenting the theca. After operation the patient was able to walk normally.

At review 1 year later, plain radiographs of the lumbar spine showed marked osteophyte formation and loss of lumbar lordosis. In 1981 the patient developed pain and weakness in the right leg. On examination she was found to have a sensory level at D8/9. A further myelogram was performed which showed no new abnormality. Her symptoms improved after the myelogram. One year later, the patient returned with further difficulty in walking associated with urinary frequency. On examination she showed a pyramidal weakness of the right leg with sensory levels at D10 on the right and L12 on the left. A lumbar myelogram revealed compression of the dorsal cord by a hypertrophied and calcified ligamentum flavum at the lower end of the previous laminectomy. Spinal CT scanning demonstrated a thickened and densely calcified posterior longitudinal ligament with associated laminal thickening (fig, b). A subsequent cervical myelogram showed a bony plaque anterior to the theca, displacing it posteriorly with compression of the cord at $\mathrm{C} 3$ and obstruction at $\mathrm{C} 4 / 5$. The patient subsequently underwent $\mathrm{C} 3$ to D1 laminectomy and further dorsal laminectomy below the site of the previous decompression. At operation the bone was found to be rock hard. The canal was narrowed in the anteroposterior diameter by a calcified posterior longitudinal ligament and thickened laminae. After operation the patient developed a quadraparesis but is now able to walk with two tripods. On examination she shows generally brisk reflexes with bilateral extensor plantar responses. There is a sensory level to approximately D8.

Case 2

A male now aged 51 years was diagnosed as having rickets 
when aged two and a half years. He was initially treated with ultraviolet light and standard doses of Vitamin D without benefit. Bilateral subtrochanteric osteotomies were performed at the age of 4 years. He began to walk aged 6 with marked bowing of his legs. He first attended this hospital in 1945 aged 12 when a diagnosis of X-linked hypophosphataemic rickets was made. He was treated with high doses of Vitamin D. Osteotomies to the left and right tibiae were performed in 1952 and 1953 respectively. He reached a final height of $4 \mathrm{ft} 8$ ins $(142 \mathrm{~cm})$.

In 1975 aged 42 he developed intermittent numbness of his legs, and 2 weeks later he experienced a sudden loss of power in his legs lasting for approximately 1 hour. This improved spontaneously. Later the same year when admitted to this hospital he was found to have a spastic paraplegia with a sensory level at D10. Plain spine radiographs then showed syndesmophytes joining the vertebral bodies from D10 downwards and calcification of the anterior and posterior spinal ligaments. Myelography was attempted by both the lumbar and cisternal roots but failed because of calcification in the spinal ligaments. The patient's condition improved spontaneously.

He returned to work and remained well until 1981 when his right arm became stiff and his legs became increasingly weak with associated flexor spasms. At that time he was found to have a spastic quadraparesis, the legs being more severely affected than the arms. Myelography showed compression of the cord at $\mathrm{C} 3 / 4$ with total obstruction at $\mathrm{C} 4 / 5$. Cloward's operation at $\mathrm{C} 3 / 4$ and $\mathrm{C} 4 / 5$ with subsequent fusion of $\mathrm{C} 3 / 4$ and 5 was performed without noticeable improvement. A second myelogram one month later showed flattening of the cord at $\mathrm{C} 3 / 4$ and occlusion of the theca at C4/5. A C3-7 laminectomy was performed. After operation the patient developed increasing weakness in the legs and a dense weakness in the right arm. Despite some improvement in these deficits, he remains unable to walk at the present time.

\section{Discussion}

Spinal cord compression associated with familial Vitamin D resistant rickets is secondary to thickening of the laminae and calcification and hypertrophy of the spinal ligaments. ${ }^{4-7}$ This complication usually occurs in affected males. In the present cases the role of calcification and hypertrophy of the spinal ligaments was clearly demonstrated by tomography and CT scanning.

The investigation of spinal cord compression by myelography may be made difficult or even impossible by the bony overgrowth and ligamentous calcification. CT scanning and presumably MRI may be valuable in such cases facilitating early diagnosis and intervention.

Our cases demonstrated a particular problem in the management of spinal cord lesions in this condition, in that compression may occur at several levels over a period of time. Both cases had experienced periods of spontaneous remission and both deteriorated significantly after surgery. In case 2 , on one occasion, an acute deterioration and subsequent rapid improvement was suggestive of an acute vascular spinal cord lesion.

\section{References}

1 Chesney RW, Mazess RB, Rose P. Supranormal 25-hydroxyvitamin D and subnormal 1,25-dihydroxyvitamin D: their role in X-linked hypophosphataemic rickets. Am J Dis Child 1980;134:140-3.

2 Albright F, Butler A, Bloomberg E. Rickets resistant to Vitamin D therapy. Am J Dis Child 1937;54:529-47.

3 Christensen JF. Three familial cases of atypical later rickets. Acta Paediatr Scand 1940;28:247-70.

4 Johnston CC Jr, Kurlander GJ, Smith DM. Familial Vitamin D rickets in untreated adult. Bony proliferation of neural arches with cord compression. Arch Int Med 1966;117:141-7.

5 Dugger GS, Vandiver RW. Spinal cord compression caused by Vitamin D resistant rickets. $J$ Neurosurg 1966;25:300-3.

6 Yoshikawa S, Shiba M, Suzuki A. Spinal cord compression in untreated adult cases of Vitamin D resistant rickets. J Bone \& Joint Surg 1968; 50-A:743-52.

7 Highman JH, Sanderson PH, Sutcliffe MM. Vitamin D resistant osteomalacia as a cause of cord compression. QJ Med 1970;39:529-37. 